\title{
Loss of fibulin-2 expression is involved in the inhibition of breast cancer invasion and forms a new barrier in addition to the basement membrane
}

\author{
HAOSHENG TAN, JIAXIN ZHANG, DEYUAN FU and YUXIANG ZHU \\ Clinical Medical School, Yangzhou University, Yangzhou, Jiangsu 225001, P.R. China \\ Received March 8, 2016; Accepted February 13, 2017
}

DOI: $10.3892 / \mathrm{ol} .2017 .6539$

\begin{abstract}
Previous studies have demonstrated that fibulin-2 may facilitate cancer cell invasion and metastasis during tumor progression. In the present study, immunohistochemical analyses of fibulin- 2 and collagen IV expression in 35 patients with breast cancer were performed to define their localization and association with breast cancer tissue. Fibulin-2 was revealed to be expressed in all tissues surrounding the breast ducts and blood vessels in normal breast tissue, while its expression was not integrated in invasive ductal carcinoma or terminal duct-lobular unit. In malignant breast tissue, collagen IV was integrated around the duct, while fibulin-2 was expressed around collagen IV and was incomplete. These results demonstrated that fibulin- 2 was associated with breast cancer invasion. Fibulin-2 expression decreased prior to basement membrane (BM) degradation, indicating that fibulin-2 forms an additional barrier around the BM. Therefore, it was proposed that fibulin-2 composes the general BM, which differs from the traditional BM. These results provide insight into extracellular matrix components and the involvement of fibulin-2 in tumor invasion and metastasis. Fibulin-2 was involved in the process of breast cancer development. It performed an important role in prevention of cancer cell penetration and metastasis.
\end{abstract}

\section{Introduction}

Breast cancer is a lethal disease with a high global incidence. For a long time, tumor cells were the only focus of cancer

Correspondence to: Dr Deyuan Fu, Clinical Medical School, Yangzhou University, 98 Nantong West Road, Yangzhou, Jiangsu 225001, P.R. China

E-mail: fdyjsyz@163.com

Abbreviations: BM, basement membrane; DCIS, ductal carcinoma in situ; ECM, extracellular matrix; IDC, invasive ductal carcinoma; TDLU, terminal duct-lobular unit

Key words: fibulin-2, breast cancer, basement membrane, extracellular matrix, invasion, metastasis studies. However, it has become clear that the extracellular matrix (ECM) performs an important function in carcinogenesis. The ECM, particularly the basement membrane (BM), acts as a barrier separating the tumor cells from the vessels which cancer metastasizes through (1-3). The procedure of tumor invasion and metastasis involves complex molecular mechanisms in cell-cell, cell-matrix and matrix-matrix interactions, which are reflected in variable up- and downregulation of multiple macromolecules (4). One of these is fibulin-2, which is associated with progression of multiple types of cancer $(5,6)$. The loss of fibulin- 2 expression results in abnormal cell adhesion and migration ability, which may facilitate cancer cell invasion and metastasis during breast cancer progression (7). In addition, previous studies have also demonstrated that fibulin-2 stabilizes the ECM in lung cancer $(8,9)$. These findings may also be due to the association of fibulin-2 expression with the progression of disease. Although fibulin-2 expression had been studied in breast cancer tissue, to the best of our knowledge there are no direct studies on the association between fibulin-2 and collagen IV, which are expressed primarily in the BM. In the present study, immunohistochemical analysis was performed to define the localization of fibulin-2 and its association with collagen IV, and the involvement of fibulin-2 and collagen IV in carcinogenesis, as well as potential therapeutic targets, were explored.

\section{Patients and methods}

Patients and tissues. Necessary consent from all patients involved in the present study was obtained, including consent to be involved in the study and consent to publish. The present study was approved by the Ethics Committees of the Clinical Medical School, Yangzhou University (Yangzhou, China). Between September 2014 and March 2015, 46 samples were collected from 23 female patients, aged 35-63. These patients included 8 ductal carcinoma in situ (DCIS) and 15 invasive ductal carcinoma (IDC). A total of two samples were collected from every patient; normal and cancer (DCIS or IDC) sections. All tissue sections were selected by an experienced pathologist from Subei People's Hospital of Jiangsu Province, Yangzhou University (Yangzhou, China), based on diagnosis and microscopic morphology. All tissues were obtained during surgical resection, and the size was $\sim 1.5 \times 0.5 \times 0.3 \mathrm{~cm}$. Antibodies used in the present study were as follows: Rabbit-anti-human fibulin-2 antibody 

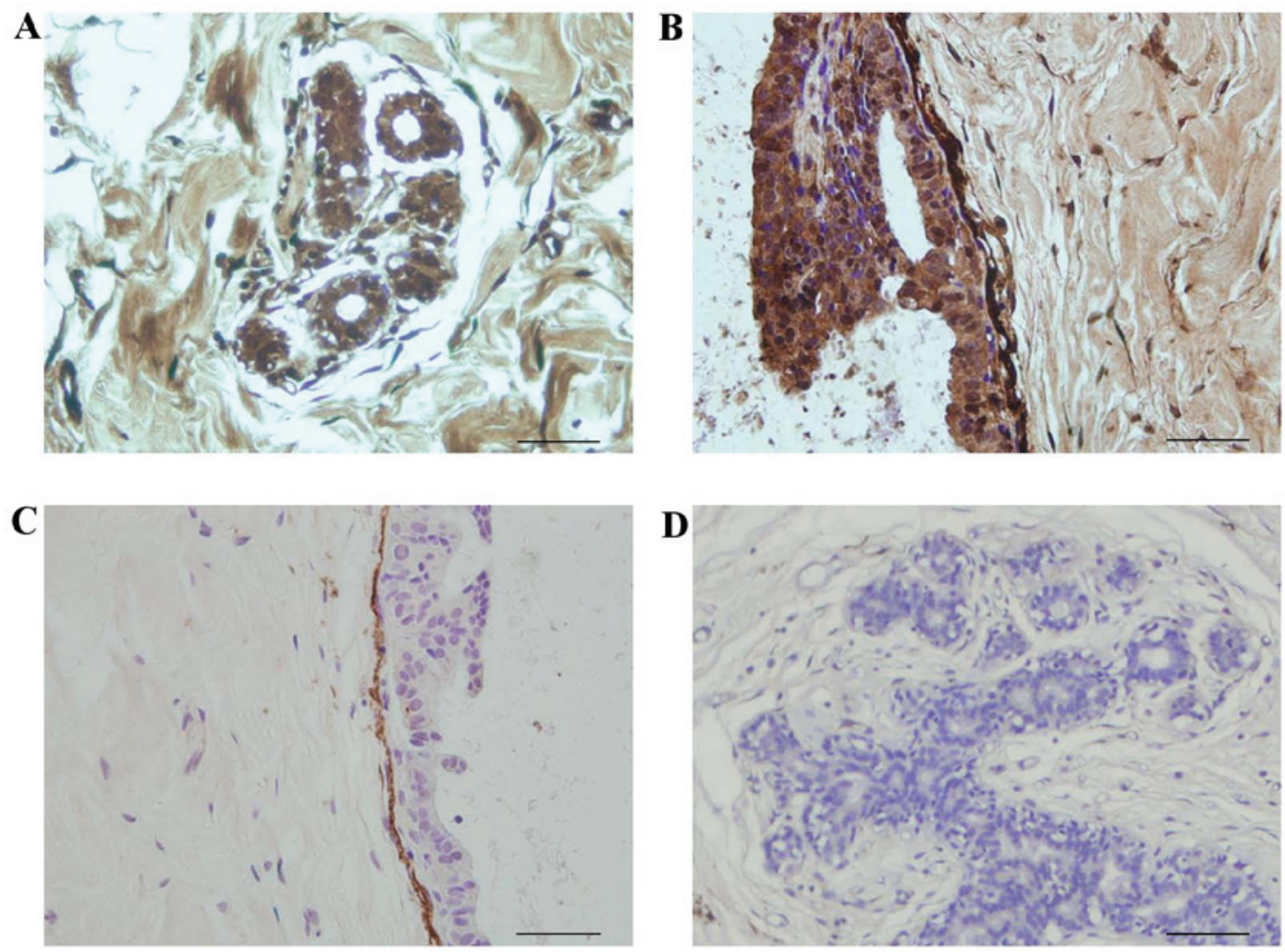

Figure 1. Expression of fibulin-2 at different antibody dilutions. (A) When the dilution of fibulin-2 antibody was 1:100, fibulin-2 appeared to express in the acinus (brown staining) and (B) around the BM (black staining). (C) When the dilution was 1:400, fibulin-2 expression was only observed around the BM (brown staining), but not in (D) the acinus. Scale bar, $25 \mu \mathrm{m}$.

(cat. no. sc-30176; Santa Cruz Biotechnology, Inc., Dallas, TX, USA); mouse-anti-human collagen IV monoclonal antibody (cat. no. MS-375; Maixin-Bio Corporation, Fuzhou, China); and MaxVison enzyme labeled goat-anti-mouse/rabbit immunoglobulin G (IgG) antibody (cat. no. KIT-5010; Maixin-Bio Corporation).

Immunohistochemical staining of fibulin-2 and collagen IV. Breast tissue samples were selected by an experienced pathologist based on diagnosis and microscopic morphology. Tissue sections $(1.5 \times 0.5 \times 0.3 \mathrm{~cm})$ were deparaffinized with xylene, and then rehydrated in ethanol washes $(100,90,80$ and $70 \%$ ). Tissues were then washed with PBS, followed by antigen retrieval through a microwave oven for 20 min (collagen IV only). Tissues were washed again with PBS and treated with $3 \% \mathrm{H}_{2} \mathrm{O}_{2}$ for 15 min to block endogenous peroxidase activity. Subsequent to washing with PBS, tissues were incubated at $24^{\circ} \mathrm{C}$ with $10 \%$ goat serum (cat. no. G9023; Sigma-Aldrich; Merck KGaA, Darmstadt, Germany) for $1 \mathrm{~h}$ to block nonspecific bindings. Samples were subsequently incubated with rabbit-anti-human fibulin-2 polyclonal antibody (at a range of dilutions, including 1:50, 1:100, 1:200 and 1:400) and mouse-anti-human collagen IV monoclonal antibody (1:400) separately overnight at $4^{\circ} \mathrm{C}$ in a humidified chamber, rinsed with PBS, and then incubated with MaxVison enzyme labeled goat-anti-mouse/rabbit IgG antibody (1:100) for $1 \mathrm{~h}$ at room temperature. Subsequent to washing with PBS, tissues were incubated for 3-5 min with 3,3'-diaminobenzidine substrate and 3-amino-9-ethylcarbazole substrate separately, followed by counterstaining with Meyer's hematoxylin for $30 \mathrm{sec}$.
Tissue sections ( $3-\mu \mathrm{m}$ thick) were mounted on coverslips, examined, and 5 fields of view were captured and assessed from every section under a light microscope (magnification range, x50-200) with a digital camera (DP70; Olympus Corporation, Tokyo, Japan).

\section{Results}

Expression of fibulin-2 at different antibody dilutions. When the dilution of fibulin-2 antibody was 1:100, fibulin-2 appeared to be expressed in the acinus (Fig. 1A) as well as around the BM (Fig. 1B). However, when the dilution was 1:400, fibulin-2 expression was only observed around the BM (Fig. 1C) and not in the acinus (Fig. 1D).

Expression of fibulin-2 and collagen IV around large breast ducts and blood vessels. Fibulin-2 was expressed around the BM outside large breast ducts (Fig. 2A) and blood vessels (Fig. 2B), while collagen IV was expressed inside the fibulin-2 layer around large breast ducts (Fig. 2C) and blood vessels (Fig. 2D).

Expression of fibulin-2 and collagen IV around medium breast ducts. Fibulin-2 was not expressed around all medium breast ducts. No fibulin-2 expression was observed around certain medium breast ducts (Fig. 3A), but fibulin-2 was expressed completely around other medium breast ducts (Fig. 3B). In adjacent breast tissue that was invaded by cancer cells, fibulin-2 was partially degraded (Fig. 3C), while collagen IV remained integrated (Fig. 3D). 

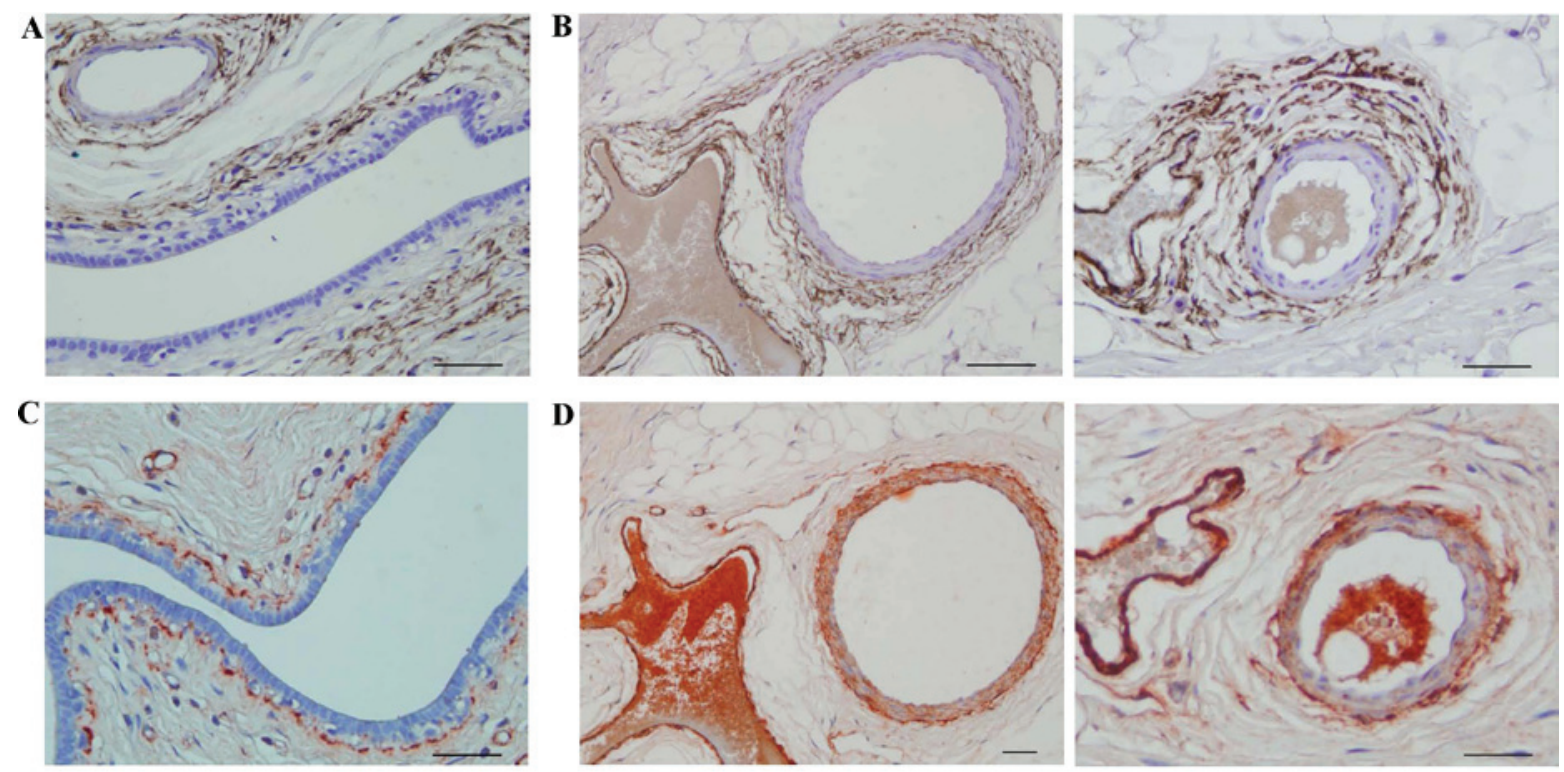

Figure 2. Expression of fibulin-2 and collagen IV around large breast ducts and blood vessels. (A) Fibulin-2 expressed around BM outside of large breast ducts (brown staining) and (B) blood vessels (brown staining), while (C) collagen IV expressed inside of fibulin-2 around large breast ducts (red staining) and (D) blood vessels (red staining). Scale bar, $25 \mu \mathrm{m}$.

$\mathbf{A}$

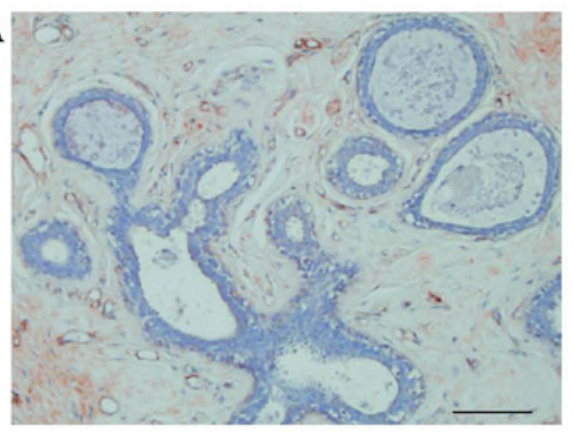

C

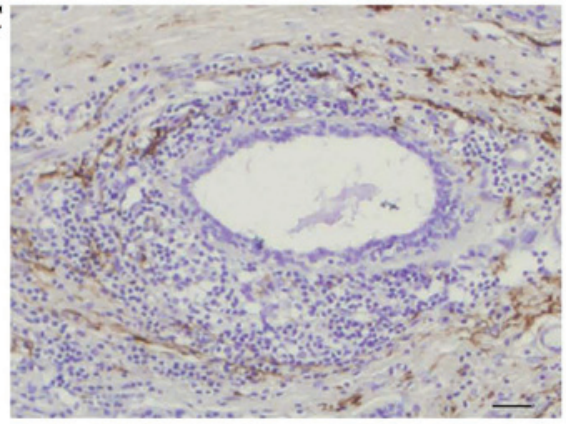

D

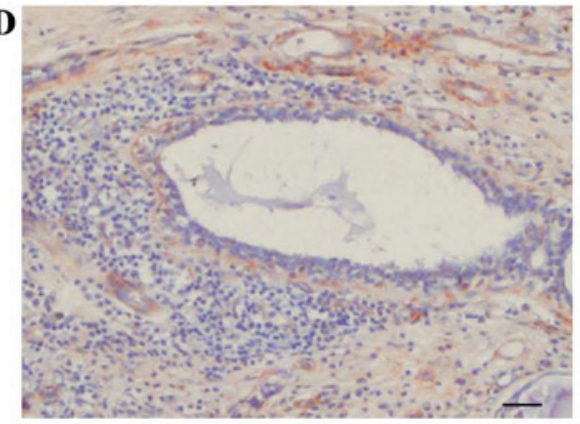

B
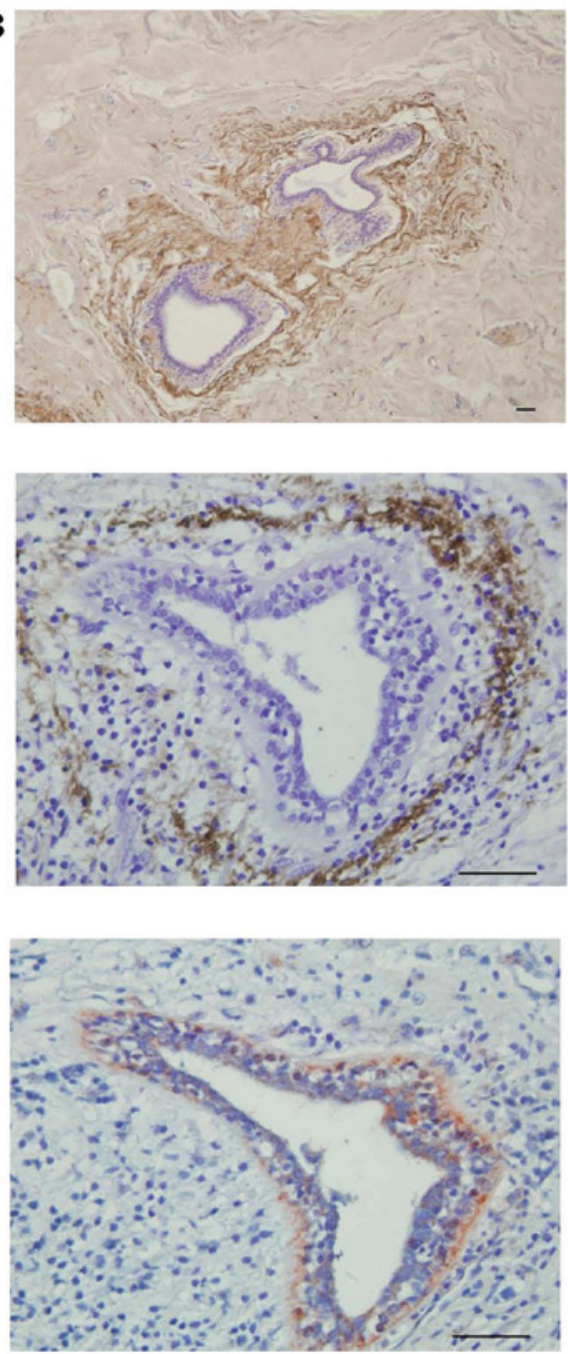

Figure 3. Expression of fibulin-2 and collagen IV around medium breast ducts. Fibulin-2 was not expressed around all medium breast ducts. (A) No fibulin-2 expression was observed around certain medium breast ducts, but (B) was expressed completely around other medium breast ducts (brown staining). (C) In adjacent breast tissue that invaded by cancer cells, fibulin-2 was partially degraded (brown staining). (D) Collagen IV remained integrated in adjacent breast tissue (red staining). Scale bar, $25 \mu \mathrm{m}$. 
$\mathbf{A}$

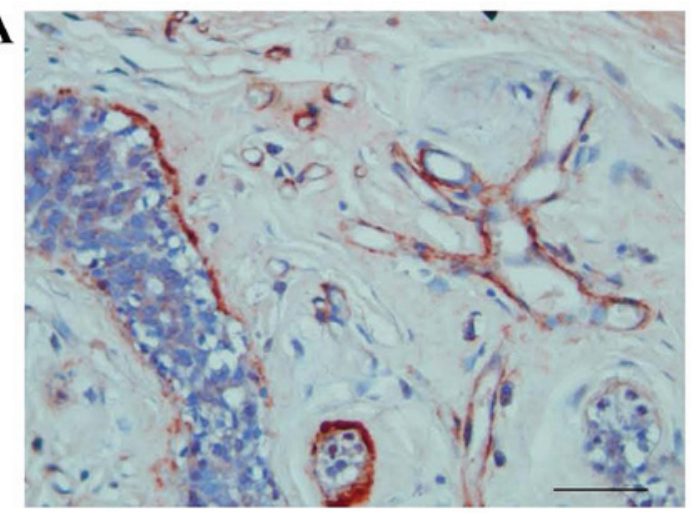

C

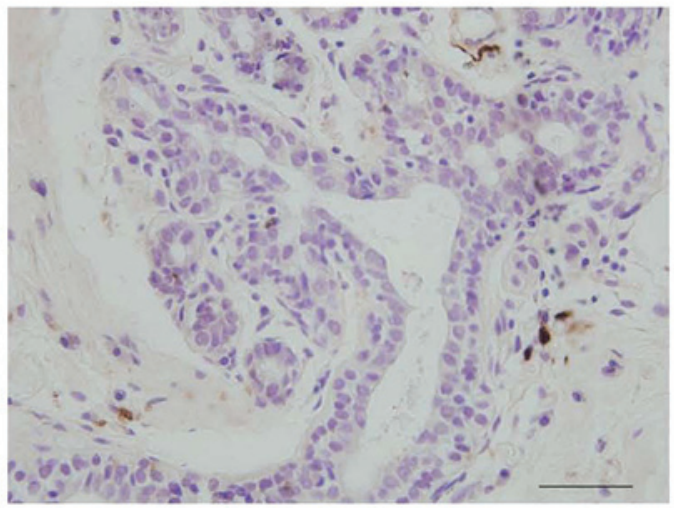

B

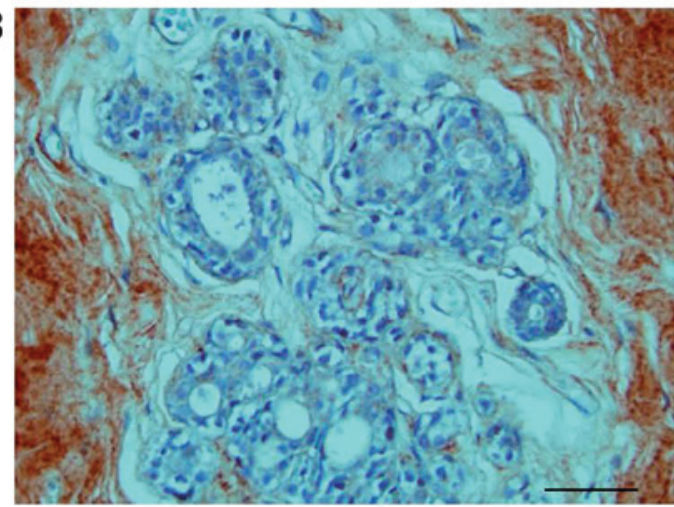

D

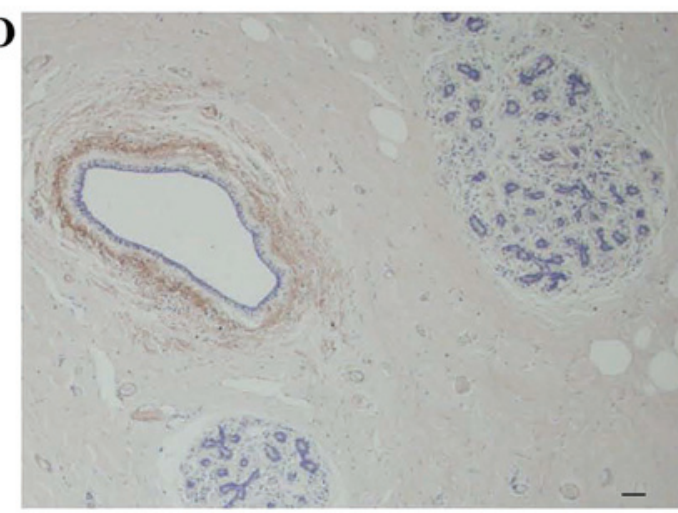

Figure 4. Expression of fibulin-2 and collagen IV in the TDLU. (A) In the TDLU, collagen IV expressed incompletely around the acinus in certain cases (red staining), (B) but expression was absent in others. (D) Fibulin-2 expression was observed around the breast ducts (brown staining), (C) but not in the acinus. Scale bar, $25 \mu \mathrm{m}$. TDLU, terminal duct-lobular unit.

Expression of fibulin-2 and collagen IV in the terminal duct-lobular unit (TDLU). In the TDLU, collagen IV was expressed incompletely around the acinus (Fig. 4A), however, occasionally expression was completely absent (Fig. 4B). Fibulin-2 was expressed around the breast ducts, but not in the acinus (Fig. 4C and D).

Expression of fibulin-2 and collagen IV in IDC. In IDC, fibulin-2 was not expressed (Fig. 5A). The expression level of collagen IV was also low (Fig. 5B).

Expression of fibulin-2 in DCIS. Fibulin-2 was expressed around certain ducts in DCIS (Fig. 6A) but not expressed around others (Fig. 6B).

\section{Discussion}

BM is widely accepted to be the first barrier of the body against cancer cells $(10,11)$. Invasion and metastasis of malignancy is a multi-step process involving multiple macromolecules. ECM contains proteins and polysaccharides synthesized and secreted by cells. The substances in the ECM form thin, dynamic sheet-like structures, involved in building tissue scaffolds and regulating embryonic development, cell migration and signal transduction $(1,2)$. A number of ECM substances regulate body functions, as well as affect the biological characteristics of tumor cells. The ECM includes BM and intercellular substances. Integrated $\mathrm{BM}$ is one of the main defense barriers inhibiting tumor invasion and metastasis. The maintenance of its normal architecture may be disrupted when the BM is invaded by tumor cells, facilitating the invasion and metastasis of these tumor cells.

Fibulin-2, which belongs to a seven-member family of extracellular glycoproteins, was first identified by Kluge et al in 1990 (12). It contains a diverse array of protein ligands, facilitating its interaction with collagen IV, fibronectin, laminin and integrin $(13,14)$. Yi et al $(7)$ reported that fibulin-2 was expressed in the cytosol and on the cell surface of normal ductal epithelial cells, particularly in the apical side of the cells. At the beginning of the immunohistochemical studies, fibulin-2 appeared to not only be expressed in mammary glandular cells and fibroblasts, but also around vessels and large breast ducts (fibulin-2 dilution was 1:100). However, when the antibody dilution was changed to $1: 400$, no fibulin-2 expression was observed in the cytosol and cell surface. $\mathrm{Gu}$ et al (15) also identified that fibulin-2 was expressed in ECM, but not in epithelial cells. Therefore, it was inferred that the immunostaining in the previous study by Yi et al (7) was non-specific. Additional innumohistochemical analysis performed in the present study demonstrated that fibulin-2 was expressed around the outside of collagen IV, with the two of them expressed around vessels and large breast ducts. In one breast tissue observed during the present study, in which ducts were invaded by cancer cells, fibulin-2 was partially degraded while collagen IV remained integrated. These findings indicated that fibulin-2 may form a new barrier outside the $\mathrm{BM}$ to defend cancer cells. In addition, fibulin- 2 was only 
$\mathbf{A}$

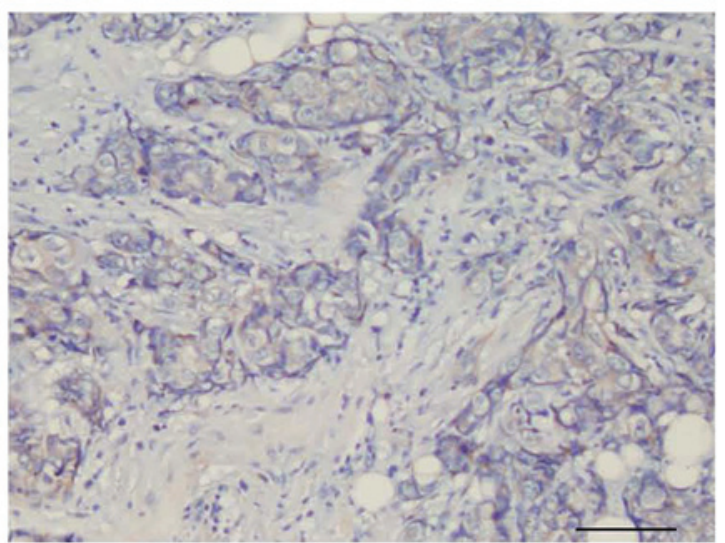

B

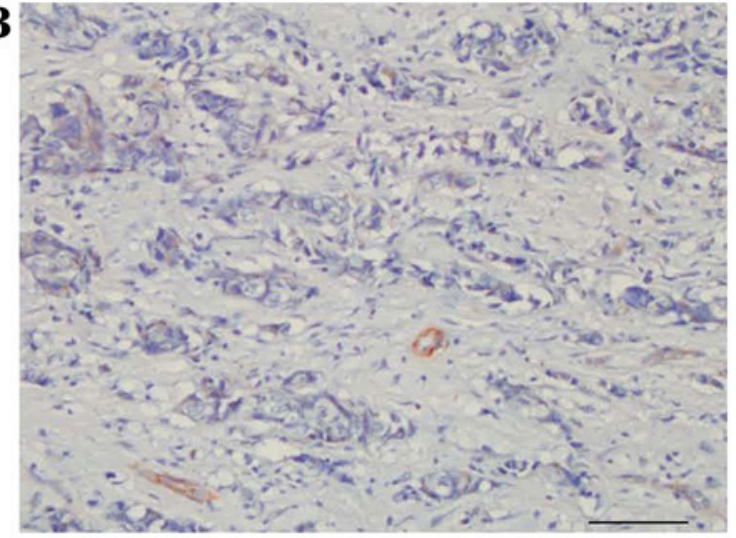

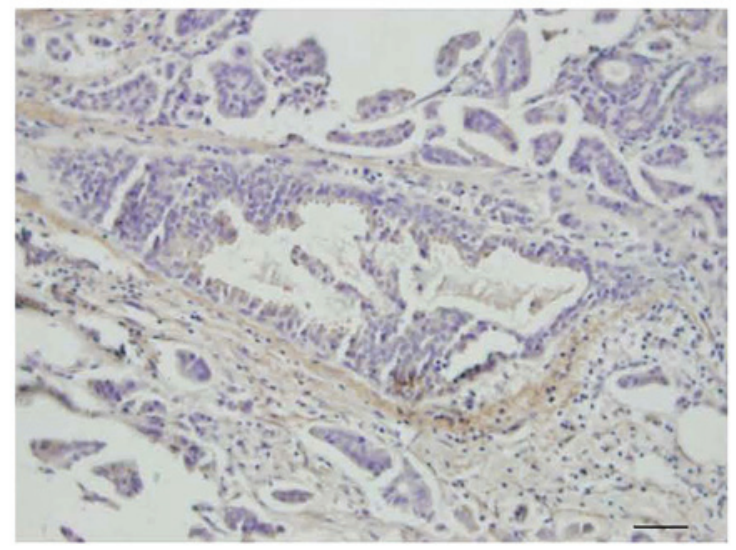

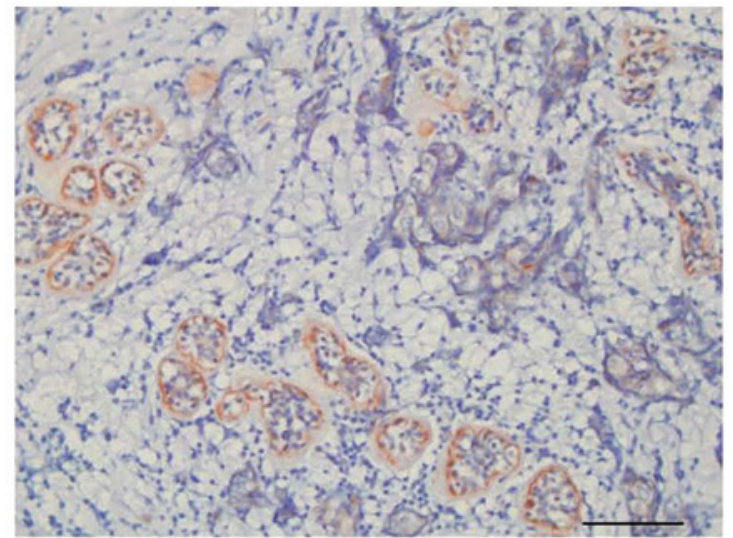

Figure 5. Expression of fibulin-2 and collagen IV in IDC. (A) In IDC, fibulin-2 was unexpressed. (B) The expression level of collagen IV was also low (brown staining). Scale bar, $25 \mu \mathrm{m}$. IDC, invasive ductal carcinoma.
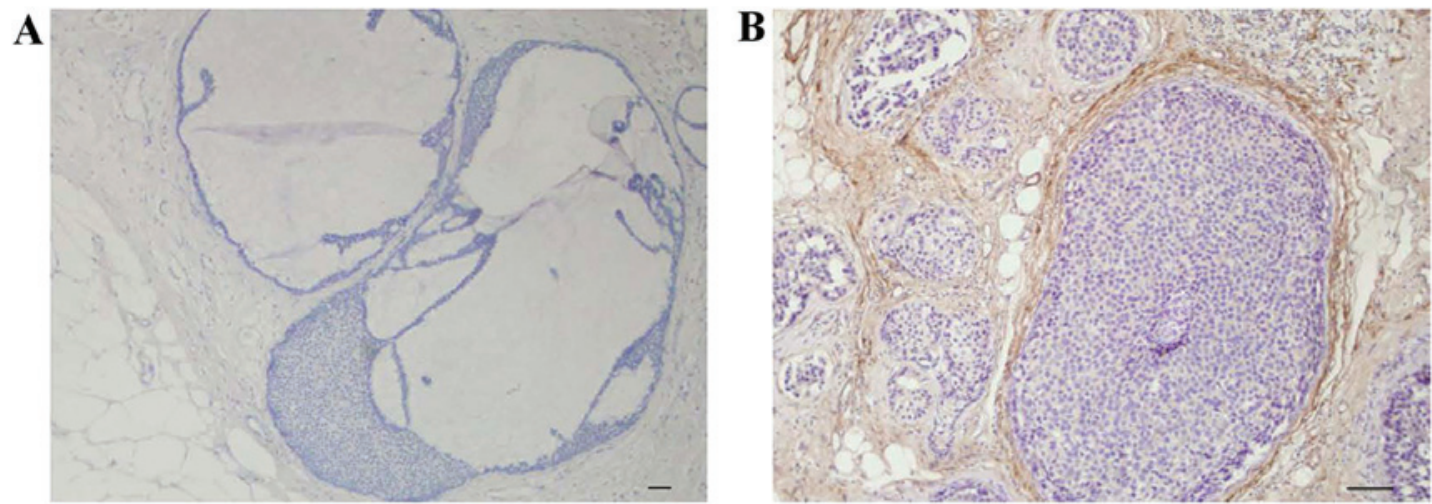

Figure 6. Expression of fibulin-2 in DCIS. (B) Fibulin-2 was expressed around certain ducts in DCIS (brown staining), but (A) was unexpressed around others. Scale bar, $25 \mu \mathrm{m}$. DCIS, ductal carcinoma in situ.

expressed partially around ducts in DCIS, with total absence of fibulin-2 observed in IDC. Yue et al (16) identified that fibulin-5 suppresses lung cancer invasion by inhibiting matrix metalloproteinase-7 expression. Therefore, it was inferred that fibulin-2 may be involved in passive defense, in which it may be degraded by matrix metalloproteinases. These results indicated that fibulin-2 is a negative regulator of invasiveness in breast cancer, and additional studies are required for its therapeutic applications in the treatment of breast cancer.

The present findings revealed that fibulin-2 is involved in breast cancer invasion and that it collapsed prior to the infiltration of the BM, indicating that it forms a barrier similar to the traditional BM. Therefore, it was hypothesized that fibulin-2 was part of the general BM, which differs from the traditional BM. These findings provide novel insight into extracellular matrix components, elucidating the involvement of fibulin-2 in tumor invasion and metastasis.

\section{Acknowledgements}

The present study was supported by the National Natural Science Foundation of China (grant no. 81172508). 


\section{References}

1. Bonnans C, Chou J and Werb Z: Remodelling the extracellular matrix in development and disease. Nat Rev Mol Cell Biol 15: 786-801, 2014.

2. Pickup MW, Mouw JK and Weaver VM: The extracellular matrix modulates the hallmarks of cancer. EMBO Rep 15: 1243-1253, 2014.

3. Salvatore V, Focaroli S, Teti G, Mazzotti A and Falconi M: Changes in the gene expression of co-cultured human fibroblast cells and osteosarcoma cells: The role of microenvironment. Oncotarget 6: 28988-28998, 2015.

4. Brady-Kalnay SM: Molecular mechanisms of cancer cell-cell interactions: Cell-cell adhesion-dependent signaling in the tumor microenvironment. Cell Adh Migr 6: 344-345, 2012.

5. Law EW, Cheung AK, Kashuba VI, Pavlova TV, Zabarovsky ER, Lung HL, Cheng Y, Chua D, Lai-Wan Kwong D, Tsao SW, et al: Anti-angiogenic and tumor-suppressive roles of candidate tumor-suppressor gene, Fibulin-2, in nasopharyngeal carcinoma. Oncogene 31: 728-738, 2012.

6. Alcendor DJ, Knobel S, Desai P, Zhu WQ and Hayward GS: KSHV regulation of fibulin-2 in Kaposi's sarcoma: Implications for tumorigenesis. Am J Pathol 179: 1443-1454, 2011.

7. Yi CH, Smith DJ, West WW and Hollingsworth MA: Loss of fibulin-2 expression is associated with breast cancer progression. Am J Pathol 170: 1535-1545, 2008.

8. Baird BN: Fibulin-2 stabilizes tumor extracellular matrix and drives malignant progression of lung adenocarcinoma (unpublished $\mathrm{PhD}$ dissertation). The University of Texas, 297, 2012.

9. Baird BN, Schliekelman MJ, Ahn YH, Chen Y, Roybal JD, Gill BJ, Mishra DK, Erez B, O'Reilly M, Yang Y, et al: Fibulin-2 is a driver of malignant progression in lung adenocarcinoma. PLoS One 8: e67054, 2013.
10. Hasengaowa, Kodama J, Kusumoto T, Shinyo Y, Seki N, Nakamura K, Hongo A and Hiramatsu Y: Loss of basement membrane heparan sulfate expression is associated with tumor progression in endometrial cancer. Eur J Gynaecol Oncol 26: 403-406, 2005.

11. Wilson DF, Jiang DJ, Pierce AM and Wiebkin OW: Oral cancer: Role of the basement membrane in invasion. Aust Dent J 44: 93-97, 1999.

12. Kluge M, Mann K, Dziadek M and Timpl R: Characterization of a novel calcium-binding 90-kDa glycoprotein (BM-90) shared by basement membranes and serum. Eur J Biochem 193: 651-659, 1990.

13. Olijnyk D, Ibrahim AM, Ferrier RK, Tsuda T, Chu ML, Gusterson BA, Stein T and Morris JS: Fibulin-2 is involved in early extracellular matrix development of the outgrowing mouse mammary epithelium. Cell Mol Life Sci 71: 3811-3828, 2014.

14. Longmate WM, Monichan R, Chu ML, Tsuda T, Mahoney MG and DiPersio CM: Reduced fibulin-2 contributes to loss of basement membrane integrity and skin blistering in mice lacking integrin $\alpha 3 \beta 1$ in the epidermis. J Invest Dermatol 134: 1609-1617, 2014.

15. Gu YC, Nilsson K, Eng $\mathrm{H}$ and Ekblom M: Association of extracellular matrix proteins fibulin-1 and fibulin-2 with fibronectin in bone marrow stroma. Br J Haematol 109: 305-313, 2000.

16. Yue W, Sun Q, Landreneau R, Wu C, Siegfried JM, Yu J and Zhang L: Fibulin-5 suppresses lung cancer invasion by inhibiting matrix metalloproteinase-7 expression. Cancer Res 69: 6339-6346, 2009. 